\title{
Tropical decomposition of Young's partition lattice
}

\author{
Vivek Dhand
}

Received: 20 December 2012 / Accepted: 1 August 2013 / Published online: 30 August 2013

(C) Springer Science+Business Media New York 2013

\begin{abstract}
Young's partition lattice $L(m, n)$ consists of integer partitions having $m$ parts where each part is at most $n$. Using methods from complex algebraic geometry, R. Stanley proved that this poset is rank-symmetric, unimodal, and strongly Sperner. Moreover, he conjectured that it has a symmetric chain decomposition, which is a stronger property. Despite many efforts, this conjecture has only been proved for $\min (m, n) \leq 4$. In this paper, we decompose $L(m, n)$ into level sets for certain tropical polynomials derived from the secant varieties of the rational normal curve in projective space, and we find that the resulting subposets have an elementary raising and lowering algorithm. As a corollary, we obtain a symmetric chain decomposition for the subposet of $L(m, n)$ consisting of "sufficiently generic" partitions.
\end{abstract}

Keywords Young's partition lattice $\cdot$ Hankel matrices · Tropical polynomials · Symmetric chain decomposition

\section{Introduction}

\subsection{Stanley's conjecture}

Young's partition lattice $L(m, n)$ is defined to be the poset of integer partitions $\lambda=$ $\left(0 \leq \lambda_{1} \leq \cdots \leq \lambda_{m} \leq n\right)$ equipped with the following partial order:

$$
\lambda \leq \mu \quad \Longleftrightarrow \quad \lambda_{i} \leq \mu_{i} \text { for all } 1 \leq i \leq m
$$

We can visualize the elements of this poset as Young diagrams that fit in the bottom left-hand corner of an $(m \times n)$ rectangle, ordered by inclusion (Fig. 1). Note that $L(m, n)$ is a ranked poset, where the rank of $\lambda$ is given by $\lambda_{1}+\cdots+\lambda_{m}$.

V. Dhand $(\varangle)$

Boulder, CO, USA

e-mail: vivek.dhand@gmail.com 


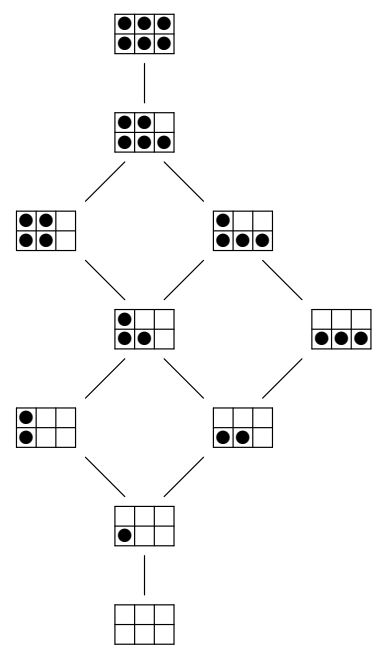

Fig. 1 The Hasse diagram of $L(2,3)$

This poset appears in different guises in several branches of mathematics. For example, it is isomorphic to the poset of Schubert cells in the Grassmannian of $m$ planes in $\mathbb{C}^{m+n}$. In the groundbreaking paper [6], R. Stanley used the hard Lefschetz theorem to prove that $L(m, n)$ is rank-symmetric, unimodal, and strongly Sperner. Furthermore, he conjectured that it has a symmetric chain decomposition, i.e. can be expressed as a disjoint union of rank-symmetric, saturated chains. A poset which has at least one symmetric chain decomposition is called a symmetric chain order. Despite many efforts, this conjecture has only been proved for $\min (m, n) \leq 4[2,5,9]$. In fact, even the problem of finding an explicit matching between adjacent ranks of $L(m, n)$ remains unsolved [4]. It is worth noting that K. O'Hara proved a weaker result by constructing a symmetric chain decomposition of the poset with the same underlying set as $L(m, n)$ but with all possible covering relations between adjacent ranks $[3,10]$.

In elementary terms, the problem is to find a rule such that:

1. For each Young diagram in $L(m, n)$, we either do nothing or remove a box so that the result is another Young diagram.

2. Each Young diagram has at most one preimage under this rule.

3. Each terminal Young diagram has complementary rank with the corresponding initial Young diagram.

The main result of this paper involves a simple algorithm which, among other things, yields a symmetric chain decomposition for a large subposet of $L(m, n)$ consisting of "sufficiently generic" partitions.

1.2 The monomial model of Young's partition lattice

Note that a partition $\lambda \in L(m, n)$ is uniquely determined by the $(n+1)$-tuple $\left(a_{0}, \ldots, a_{n}\right)$ where $a_{i}$ is equal to the number of times that $i$ appears in $\left(\lambda_{1}, \ldots, \lambda_{m}\right)$. 
A covering relation in $L(m, n)$ is given by adding a box to an acceptable row of a Young diagram, which sends

$$
\left(a_{0}, \ldots, a_{n}\right) \mapsto\left(a_{0}, \ldots, a_{i-1}-1, a_{i}+1, \ldots, a_{n}\right) .
$$

In this way, we obtain an edge coloring of the Hasse diagram of $L(m, n)$, using $n$ colors, where the $i$ th color corresponds to the above operation.

Let $A_{n}=\mathbb{C}\left[z_{0}, \ldots, z_{n}\right]$. By mapping $\left(a_{0}, \ldots, a_{n}\right)$ to the monomial $z_{0}^{a_{0}} \ldots z_{n}^{a_{n}}$ we see that $L(m, n)$ is isomorphic to the poset $A_{n}(m)$ of monomials of degree $m$ in $A_{n}$. In terms of partitions, this isomorphism is given by

$$
\left(\lambda_{1}, \ldots, \lambda_{m}\right) \mapsto z_{\lambda_{1}} z_{\lambda_{2}} \cdots z_{\lambda_{m}}
$$

The $i$ th color operation corresponds to changing a single $z_{i-1}$ to $z_{i}$, and this partial order is induced by the standard action of $\mathfrak{s l}_{2} \mathbb{C}$ on the irreducible representation $\mathbb{C}\left\langle z_{0}, \ldots, z_{n}\right\rangle$. The advantage of using this model for Young's partition lattice is that we can simultaneously deal with $A_{n}(m)$ for all $m \geq 0$ and exploit the full structure of $A_{n}$ as a commutative graded $\mathbb{C}$-algebra with $\mathfrak{s l}_{2} \mathbb{C}$-action.

\subsection{Combinatorial secant ideals of the rational normal curve}

Since finding a symmetric chain decomposition of $L(m, n)$ is so difficult, we might first look for a coarser decomposition into centered subposets. From the point of view of commutative algebra, a natural way to decompose the monomial basis in $A_{n}$ is to choose a monomial ideal $I \subset A_{n}$ and form the graded algebra:

$$
\operatorname{gr}_{I}\left(A_{n}\right)=\bigoplus_{j \geq 0} I^{j} / I^{j+1} \text {. }
$$

In fact, we can obtain even finer decompositions by starting with a set of monomial ideals $I_{1}, \ldots, I_{t} \subset A_{n}$ and looking at the multigraded algebra

$$
\operatorname{gr}_{I_{1}} \cdots \operatorname{gr}_{I_{t}} A_{n}
$$

In order to take full advantage of the underlying symmetry, we should start with a set of $\mathfrak{s l}_{2} \mathbb{C}$-invariant ideals and perform a Gröbner degeneration to obtain monomial ideals.

For the rest of the paper, let $k=\lfloor n / 2\rfloor$. From the point of view of algebraic geometry, the nicest possible $\mathfrak{s l}_{2} \mathbb{C}$-invariant subvarieties of projective space are the rational normal curve $\mathcal{C}_{n} \subset \mathbb{P}^{n}$ and its secant varieties $\mathcal{C}_{n}^{\{r\}}$ for $0 \leq r \leq k$. By considering the initial ideals of $\mathcal{C}_{n}^{\{r\}}$ (with respect to any diagonal term order) we obtain a set of squarefree monomial ideals $I_{n, r} \subset A_{n}$ for $0 \leq r \leq k$.

We can now equip the monomial basis in $A_{n}$ with extra gradings according to the order of vanishing on this set of monomial ideals. Given a monomial $\mu \in A_{n}$, let $\operatorname{deg}_{r}(\mu)$ be the number of minimal generators of $I_{n, r}$ appearing in the image of $\mu$ under the natural map

$$
A_{n} \rightarrow \operatorname{gr}_{I_{n, k}} \cdots \operatorname{gr}_{I_{n, 1}} A_{n}
$$


Given integers $d_{0}, \ldots, d_{k} \geq 0$, we define

$$
Q_{n}\left(d_{0}, \ldots, d_{k}\right)=\left\{\mu \in A_{n} \mid \operatorname{deg}_{r}(\mu)=d_{r} \text { for each } 0 \leq r \leq k\right\} .
$$

Set-theoretically, this decomposition is implicit in Conca's "canonical decomposition" algorithm for factoring monomials [1]. However, our description (in terms of tropical polynomials, see below) does not depend on a particular choice of factorization and therefore allows for a finer investigation of the poset structure.

It turns out that $I_{n, 1}$ is the Stanley-Reisner ideal of the path graph with vertices $\{0, \ldots, n\}$, and $I_{n, r}$ is the $r$ th combinatorial secant ideal of $I_{n, 1}[7,8]$. Using these facts, we find an explicit formula for the irredundant irreducible decomposition of each $I_{n, r}$, which leads us to the following tropical polynomials:

$$
f_{n, r}\left(a_{0}, \ldots, a_{n}\right)=\min _{0 \leq \lambda_{0} \leq \cdots \leq \lambda_{n-2 r} \leq r} \sum_{j=0}^{n-2 r} a_{2 \lambda_{j}+j}
$$

For any monomial $\mu=z_{0}^{a_{0}} \cdots z_{n}^{a_{n}}$, we define $f_{n, r}(\mu)=f_{n, r}\left(a_{0}, \ldots, a_{n}\right)$. We have:

$$
\mu \in I_{n, r}^{(s)} \quad \Longleftrightarrow \quad f_{n, r}(\mu) \geq s,
$$

where $I^{(s)}$ denotes the $s$ th symbolic power of $I$. Remarkably, grading by the symbolic powers give us the same decomposition of monomials as the ordinary powers:

$$
Q_{n}\left(d_{0}, \ldots, d_{k}\right)=\left\{\mu \in A_{n} \mid f_{n, r}(\mu)=\sum_{j=r}^{k}(j+1-r) d_{j} \text { for each } 0 \leq r \leq k\right\} .
$$

With this explicit description in hand, we find that $Q_{n-2}\left(d_{1}, \ldots, d_{k}\right)$ embeds into $Q_{n}\left(d_{0}, \ldots, d_{k}\right)$ in two opposite ways, and there exists an elementary algorithm for producing coverings by saturated chains running between these two extremes.

\subsection{The raising and lowering algorithm}

A pair of consecutive entries $\left(a_{i}, a_{i+1}\right)$ in $\left(a_{0}, \ldots, a_{n}\right)$ is called a maximal pair if

$$
a_{i}+a_{i+1}=\max _{0 \leq j \leq n-1}\left(a_{j}+a_{j+1}\right)
$$

Let us now describe the crucial algorithm that produces saturated chains in $Q_{n}\left(d_{0}\right.$, $\left.\ldots, d_{k}\right)$ running between the two extremal copies of $Q_{n-2}\left(d_{1}, \ldots, d_{k}\right)$.

\section{The algorithm (right-moving version)}

1. Choose a maximal pair $\left(a_{i}, a_{i+1}\right)$ for some $0 \leq i \leq n-1$. If $i=n-1$, apply the $n$th color $a_{n-1}$ times and end the chain.

2. Otherwise, apply the $(i+1)$ th color $\left(a_{i}-a_{i+2}\right)$ times, and then go back to the first step and start with the maximal pair $\left(a_{i+1}, a_{i+2}\right)$. 


\section{The algorithm (left-moving version)}

1. Choose a maximal pair $\left(a_{i-1}, a_{i}\right)$ for some $1 \leq i \leq n$. If $i=1$, apply the inverse of the first color $a_{1}$ times and end the chain.

2. Otherwise, apply the inverse of the $i$ th color $\left(a_{i}-a_{i-2}\right)$ times, and then go back to the first step and start with the maximal pair $\left(a_{i-2}, a_{i-1}\right)$.

Given a monomial $\mu=z_{0}^{a_{0}} \cdots z_{n}^{a_{n}} \in Q_{n}\left(d_{0}, \ldots, d_{k}\right)$, we apply the two versions of the algorithm to the leftmost (resp. rightmost) maximal pair in $\left(a_{0}, \ldots, a_{n}\right)$ to obtain the left (resp. right) transversal chain of $\mu$.

Theorem Let $n, d_{0}, \ldots, d_{k} \geq 0$. The transversal chains provide a vertex covering of $Q_{n}\left(d_{0}, \ldots, d_{k}\right)$ by saturated chains. If $d_{0}>0$, then this covering is actually a decomposition.

Transversal chains are not necessarily rank-symmetric. Nevertheless, when dealing with sufficiently generic monomials, we can stitch them together to get a symmetric chain decomposition.

Corollary If $Q_{n-2}\left(d_{1}, \ldots, d_{k}\right)$ has a symmetric chain decomposition and

$$
1+2 d_{0} \geq \sum_{i=1}^{k} d_{i}(n-2 i) i
$$

then $Q_{n}\left(d_{0}, \ldots, d_{k}\right)$ has a symmetric chain decomposition.

In other words, we can decompose Young's partition lattice into a "generic" part and a "singular" part:

$$
L(m, n)=L(m, n)^{\mathrm{gen}} \sqcup L(m, n)^{\mathrm{sing}},
$$

where $L(m, n)^{\text {gen }}$ is a symmetric chain order, and $L(m, n)^{\text {sing }}$ has a covering by transversal chains. Note that by a decomposition of a poset we mean a set-theoretic decomposition where each factor is equipped with the induced partial order.

Let us outline the contents of the paper. In Sect. 2, we decompose $L(m, n)$ according to the order of vanishing on the monomial ideals $I_{n, r}$ for $0 \leq r \leq k$, and we give a simpler description in terms of the level sets of certain tropical polynomials. The poset structure aside, the results of this section are essentially contained in [1]. In Sect. 3, we use the tropical description to prove that each level set in $A_{n}$ contains two extremal embeddings of a smaller level set in $A_{n-2}$. In Sect. 4, we prove that the raising and lowering algorithm yields a covering of the level sets by monotonic saturated chains running between the extremal subposets. Indeed, most of the time this covering is a decomposition, and this fact allows us to inductively stitch these chains together to obtain a symmetric chain decomposition for the generic part of $L(m, n)$. 


\section{Combinatorial secant ideals of the rational normal curve}

Let $A_{n}=\mathbb{C}\left[z_{0}, \ldots, z_{n}\right]$, and let $A_{n}(m)$ denote the set of monomials of degree $m$ in $A_{n}$. Define a partial order on $A_{n}(m)$ as follows:

$$
z_{0}^{a_{0}} \cdots z_{n}^{a_{n}} \leq z_{0}^{b_{0}} \cdots z_{n}^{b_{n}} \Longleftrightarrow \sum_{i=j}^{n} a_{i} \leq \sum_{i=j}^{n} b_{i} \quad \text { for } 1 \leq j \leq n .
$$

This partial order is induced by the following action of $\mathfrak{s l}_{2} \mathbb{C}=\mathbb{C}\langle H, E, F\rangle$ on the irreducible representation $\mathbb{C}\left\langle z_{0}, \ldots, z_{n}\right\rangle$ :

$$
H\left(z_{i}\right)=n-2 i, \quad E\left(z_{i}\right)=i z_{i-1}, \quad F\left(z_{i}\right)=(n-i) z_{i+1} .
$$

It is not hard to see that $L(m, n)$ is isomorphic to $A_{n}(m)$ by the map

$$
\left(\lambda_{1}, \ldots, \lambda_{m}\right) \mapsto z_{\lambda_{1}} z_{\lambda_{2}} \cdots z_{\lambda_{m}}
$$

Note that $L(m, n) \simeq L(n, m)$ simply by taking the conjugate partition, which corresponds to the well-known duality $A_{n}(m) \simeq A_{m}(n)$ for choosing multisets from a finite set. Also note that the weight of a monomial $\mu=z_{0}^{a_{0}} \cdots z_{n}^{a_{n}}$ is given by

$$
\mathrm{wt}(\mu)=\sum_{i=0}^{n} a_{i}(n-2 i)
$$

while its rank is given by

$$
\operatorname{rk}(\mu)=\sum_{i=0}^{n} i a_{i}
$$

and therefore we obtain the simple relation

$$
\operatorname{wt}(\mu)=m n-2 \operatorname{rk}(\mu) .
$$

Since we will be working exclusively with centered subposets of $A_{n}(m)$, it will be more convenient for us to refer to the weights of monomials instead of their ranks.

Recall that $k:=\lfloor n / 2\rfloor$. The ideal of the rational normal curve $\mathcal{C}_{n} \subset \mathbb{P}^{n}$ is generated by the set of maximal minors of the $(2 \times n)$-Hankel matrix:

$$
H_{n, 1}=\left[\begin{array}{cccc}
z_{0} & z_{1} & \cdots & z_{n-1} \\
z_{1} & z_{2} & \cdots & z_{n}
\end{array}\right]
$$

Furthermore, the ideal of the $r$ th secant variety of $\mathcal{C}_{n}$ is generated by the set of maximal minors of the $(r+1) \times(n-r+1)$ Hankel matrix

$$
H_{n, r}=\left[\begin{array}{cccc}
z_{0} & z_{1} & \ldots & z_{r} \\
z_{1} & z_{2} & \ldots & z_{r+1} \\
\vdots & \vdots & & \vdots \\
z_{n-r} & z_{n-r+1} & \ldots & z_{n}
\end{array}\right]
$$


where $1 \leq r \leq k$. Let $I_{n, r}$ denote the initial ideal (with respect to any diagonal term order) of the ideal of the $r$ th secant variety of $\mathcal{C}_{n}$. The minimal generators of $I_{n, r}$ are the initial monomials of Hankel determinants,

$$
I_{n, r}=\left(\left\{z_{i_{0}} \cdots z_{i_{r}} \mid i_{j}+1<i_{j+1} \text { for } 0 \leq j \leq r-1\right\}\right) .
$$

For ease of notation, we define $I_{n}=I_{n, 1}$ and $I_{n, 0}=\mathfrak{m}=\left(z_{0}, \ldots, z_{n}\right)$.

Recall that for an ideal $I$ in a ring $A$ and an element $a \in A$ such that

$$
a \notin \bigcap_{j \geq 0} I^{j},
$$

the order of vanishing of a on $I$ is defined as

$$
\operatorname{ord}_{I}(a)=\max \left\{j \mid a \in I^{j}\right\} .
$$

The associated graded ring

$$
\operatorname{gr}_{I} A=\bigoplus_{j \geq 0} I^{j} / I^{j+1}
$$

receives a natural set map

$$
\sigma: A \rightarrow \operatorname{gr}_{I} A
$$

where

$$
\begin{aligned}
& \sigma(a)=a\left(\bmod I^{d+1}\right) \text { if } \operatorname{ord}_{I}(a)=d, \\
& \sigma(a)=0 \quad \text { if } a \in \bigcap_{j \geq 0} I^{j} .
\end{aligned}
$$

Also, if $J \subset A$ is another ideal, we can form the multigraded algebra

$$
\operatorname{gr}_{J} \operatorname{gr}_{I} A:=\operatorname{gr}_{\widetilde{J}} \operatorname{gr}_{I} A
$$

where $\widetilde{J}$ denotes the ideal generated by $\sigma(J) \subset \operatorname{gr}_{I}(A)$.

In our situation, we will consider the composition of several such maps:

$$
A_{n} \rightarrow \operatorname{gr}_{I_{n, 1}} A_{n} \rightarrow \operatorname{gr}_{I_{n, 2}} \operatorname{gr}_{I_{n, 1}} A_{n} \rightarrow \cdots \rightarrow \operatorname{gr}_{I_{n, k}} \ldots \operatorname{gr}_{I_{n, 1}} A_{n}
$$

Given a monomial $\mu \in A_{n}$, let $\operatorname{deg}_{r}(\mu)$ be the number of minimal generators of $I_{n, r}$ appearing in the image of $\mu$ under the above composition. A maximal factorization of $\mu$ is any factorization of $\mu$ in $A_{n}$ of the form

$$
\mu=\mu_{0} \mu_{1} \cdots \mu_{k}
$$

where each $\mu_{r}$ is a product of $\operatorname{deg}_{r}(\mu)$ minimal generators of $I_{n, r}$.

We can visualize a maximal factorization as a tableau with $\operatorname{deg}_{r}(\mu)$ rows of length $(r+1)$, where the entries in each row form a $<1$-chain, i.e., a sequence of nonnegative integers $\left(i_{0}, \ldots, i_{r}\right)$ such that $i_{j}+1<i_{j+1}$ for $0 \leq j \leq r-1$. Let us rearrange 
the entries in this tableau so that each entry is as small as possible as we read from left to right and from the longest to the shortest row. This is the same tableau obtained by factoring out each minimal generator of $I_{n, r}$ in lexicographic order as $r$ runs from $k$ to 0 , which is exactly Conca's algorithm for "canonical decomposition" of monomials [1]. The key property of Conca's tableau is that, for any entry $a$, either $a$ or $a-1$ must appear in each preceding row. Otherwise, we could insert $a$ at an earlier place in the tableau while preserving the $<_{1}$-chains along the rows, which would contradict the fact that the tableau is initial for the lexicographic order.

Example 2.1 The monomial $\mu=z_{0} z_{2} z_{3} z_{4} z_{5} \in A_{5}(5)$ has two possible maximal factorizations:

\begin{tabular}{|l|l|l|}
\hline 0 & 2 & 4 \\
\hline 3 & 5
\end{tabular}$\quad$\begin{tabular}{|l|l|l|}
\hline 0 & 3 & 5 \\
\hline 2 & 4 & \multicolumn{1}{|c}{} \\
\hline
\end{tabular}

The one on the left is Conca's tableau for $\mu$.

Definition 2.2 Following the above discussion, we obtain a multigraded decomposition of the monomial basis in $A_{n}$ :

$$
Q_{n}\left(d_{0}, \ldots, d_{k}\right)=\left\{\mu \in A_{n} \mid \operatorname{deg}_{r}(\mu)=d_{r} \text { for all } 0 \leq r \leq k\right\}
$$

Note that $\mu \in Q_{n}\left(d_{0}, \ldots, d_{k}\right)$ if and only if Conca's tableau for $\mu$ has exactly $d_{i}$ rows of size $(i+1)$. Also note that $Q_{n}\left(d_{0}, \ldots, d_{k}\right) \subset A_{n}(m)$, where

$$
m=\sum_{r=0}^{k} d_{r}(r+1) .
$$

While our decomposition is set-theoretically equivalent to Conca's decomposition, there are a few subtle but important differences. Conca's construction yields one particular maximal factorization for each monomial. This choice obscures the poset structure, which is our main object of interest. The goal of the rest of this section is to give a description of $Q_{n}\left(d_{0}, \ldots, d_{k}\right)$ that treats all maximal factorizations equally and illuminates the structural relationships between these posets.

To begin with, we give an explicit description of the unique irredundant irreducible decomposition of each $I_{n, r}$ in terms of the associated simplicial complex. Let $\Delta_{n}$ denote the set of subsets of $\{0, \ldots, n\}$. For any $F \in \Delta_{n}$, we define

$$
z^{F}=\prod_{i \in F} z_{i} \in A_{n} \quad \text { and } \quad \mathfrak{m}^{F}=\sum_{i \in F}\left(z_{i}\right) \subset A_{n} .
$$

Let $\Gamma \subset \Delta_{n}$ be an abstract simplicial complex, so $S \subset T \in \Gamma \Longrightarrow S \in \Gamma$. The Stanley-Reisner ideal of $\Gamma$ is defined as follows:

$$
I_{\Gamma}=\left\langle z^{F} \mid F \notin \Gamma\right\rangle=\bigcap_{\bar{F} \in \Gamma} \mathfrak{m}^{F} .
$$


For our problem, the relevant simplicial complex is the path graph with $n+1$ vertices,

$$
\Gamma_{n}=\{\varnothing,\{0\}, \ldots,\{n\},\{0,1\},\{1,2\}, \ldots,\{n-1, n\}\} .
$$

It is easy to check that the Stanley-Reisner ideal of $\Gamma_{n}$ is equal to $I_{n}$. Indeed, $I_{n} \subset I_{\Gamma_{n}}$ because $I_{n}$ is generated by the quadratic monomials corresponding to the edges in the complement of the path graph. On the other hand, any $F \in \Delta_{n}$ such that $|F| \geq 3$ must contain some $i$ and $j$ such that $i+1<j$, so we see that $I_{\Gamma_{n}} \subset I_{n}$.

Remark 2.3 We know from Sect. 6.1 of [8] that $I_{n, r}$ is equal to the $r$ th combinatorial secant ideal of $I_{n}$. Let $\Gamma_{n, r}$ denote the simplicial complex of $r$-fold unions of simplices from $\Gamma_{n}$ :

$$
\Gamma_{n, r}=\left\{F_{1} \cup \cdots \cup F_{r} \mid F_{i} \in \Gamma_{n}\right\} .
$$

By Remark 2.9 of [7], it follows that $I_{n, r}$ is the Stanley-Reisner ideal of $\Gamma_{n, r}$. Furthermore, it is not hard to see that each facet of $\Gamma_{n, r}$ is equal to the disjoint union of $r$ edges in $\Gamma_{n}$. Therefore, the set of $F \in \Delta_{n}$ such that $\bar{F}$ is a facet of $\Gamma_{n, r}$ is equal to

$$
\left\{\left\{2 \lambda_{0}, 2 \lambda_{1}+1, \ldots, 2 \lambda_{n-2 r}+n-2 r\right\} \mid 0 \leq \lambda_{0} \leq \cdots \leq \lambda_{n-2 r} \leq r\right\} .
$$

It follows that the irredundant irreducible decomposition of $I_{n, r}$ (cf. [1], Lemma 3.5) is given by

$$
I_{n, r}=\bigcap_{0 \leq \lambda_{0} \leq \cdots \leq \lambda_{n-2 r} \leq r} \mathfrak{m}^{\left\{2 \lambda_{0}, 2 \lambda_{1}+1, \ldots, 2 \lambda_{n-2 r}+n-2 r\right\}} .
$$

We are now ready to describe the symbolic powers of our monomial ideals. If $I$ is a radical ideal in a polynomial ring over an algebraically closed field, then the $s$ th symbolic power of $I$ is

$$
I^{(s)}=\bigcap_{M \in \mathcal{M}_{I}} M^{s},
$$

where $\mathcal{M}_{I}$ denotes the set of all maximal ideals containing $I$ [8].

Proposition 2.4 For each $0 \leq r \leq k$, the tropical polynomial

$$
f_{n, r}\left(a_{0}, \ldots, a_{n}\right)=\min _{0 \leq \lambda_{0} \leq \cdots \leq \lambda_{n-2 r} \leq r} \sum_{j=0}^{n-2 r} a_{2 \lambda_{j}+j}
$$

satisfies the following property:

$$
z_{0}^{a_{0}} \cdots z_{n}^{a_{n}} \in I_{n, r}^{(s)} \quad \Longleftrightarrow \quad f_{n, r}\left(a_{0}, \ldots, a_{n}\right) \geq s .
$$

Proof In fact, there is a general relationship between symbolic powers and tropical polynomials. Any squarefree monomial ideal $I$ with the irredundant irreducible decomposition

$$
I=\bigcap_{F} \mathfrak{m}^{F}
$$


has the following symbolic powers:

$$
I^{(s)}=\bigcap_{F}\left(\mathfrak{m}^{F}\right)^{s}
$$

Therefore,

$$
z_{0}^{a_{0}} \cdots z_{n}^{a_{n}} \in I^{(s)} \quad \Longleftrightarrow \quad z_{0}^{a_{0}} \ldots z_{n}^{a_{n}} \in\left(\mathfrak{m}^{F}\right)^{s} \quad \text { for all } F \text {. }
$$

Now,

$$
\left(\mathfrak{m}^{F}\right)^{s}=\left(\left\{\prod_{i \in F} z_{i}^{b_{i}} \mid \sum_{i \in F} b_{i}=s\right\}\right)
$$

and so

$$
z_{0}^{a_{0}} \cdots z_{n}^{a_{n}} \in\left(\mathfrak{m}^{F}\right)^{s} \Longleftrightarrow \sum_{i \in F} a_{i} \geq s .
$$

In our particular example, we obtain:

$$
z_{0}^{a_{0}} \cdots z_{n}^{a_{n}} \in I_{n, r}^{(s)} \quad \Longleftrightarrow \quad \sum_{j=0}^{2 n-r} a_{2 \lambda_{j}+j} \geq s \quad \text { for all } 0 \leq \lambda_{0} \leq \cdots \leq \lambda_{n-2 r} \leq r,
$$

which is equivalent to $f_{n, r}\left(a_{0}, \ldots, a_{n}\right) \geq s$.

Example 2.5 If $n=5$, then the ideal

$$
I_{5,1}=\left(z_{0} z_{2}, z_{0} z_{3}, z_{0} z_{4}, z_{0} z_{5}, z_{1} z_{3}, z_{1} z_{4}, z_{1} z_{5}, z_{2} z_{4}, z_{2} z_{5}, z_{3} z_{5}\right)
$$

has the following irredundant irreducible decomposition:

$$
\left(z_{0}, z_{1}, z_{2}, z_{3}\right) \cap\left(z_{0}, z_{1}, z_{2}, z_{5}\right) \cap\left(z_{0}, z_{1}, z_{4}, z_{5}\right) \cap\left(z_{0}, z_{3}, z_{4}, z_{5}\right) \cap\left(z_{2}, z_{3}, z_{4}, z_{5}\right),
$$

and the corresponding tropical polynomial is

$$
f_{5,1}\left(a_{0}, \ldots, a_{5}\right)=\min \left\{\begin{array}{l}
a_{0}+a_{1}+a_{2}+a_{3} \\
a_{0}+a_{1}+a_{2}+a_{5} \\
a_{0}+a_{1}+a_{4}+a_{5} \\
a_{0}+a_{3}+a_{4}+a_{5} \\
a_{2}+a_{3}+a_{4}+a_{5}
\end{array}\right\} .
$$

Similarly,

$$
\begin{aligned}
I_{5,2} & =\left(z_{0} z_{2} z_{4}, z_{0} z_{2} z_{5}, z_{0} z_{3} z_{5}, z_{1} z_{3} z_{5}\right) \\
& =\left(z_{0}, z_{1}\right) \cap\left(z_{0}, z_{3}\right) \cap\left(z_{0}, z_{5}\right) \cap\left(z_{2}, z_{3}\right) \cap\left(z_{2}, z_{5}\right) \cap\left(z_{4}, z_{5}\right), \\
f_{5,2}\left(a_{0}, \ldots, a_{5}\right) & =\min \left(a_{0}+a_{1}, a_{0}+a_{3}, a_{0}+a_{5}, a_{2}+a_{3}, a_{2}+a_{5}, a_{4}+a_{5}\right) .
\end{aligned}
$$


Remark 2.6 For a monomial $\mu=z_{0}^{a_{0}} \cdots z_{n}^{a_{n}}$, note that $f_{n, r}$ minimizes a sum over the complements of facets of $\Gamma_{n, r}$ :

$$
f_{n, r}(\mu)=\min _{F \in \Gamma_{n, r}} \sum_{i \notin F} a_{i} .
$$

Since $\operatorname{deg}(\mu)=a_{0}+\cdots+a_{n}$, we can rewrite this as

$$
f_{n, r}(\mu)=\min _{F \in \Gamma_{n, r}}\left(\operatorname{deg}(\mu)-\sum_{i \in F} a_{i}\right)=\operatorname{deg}(\mu)-\max _{F \in \Gamma_{n, r}} \sum_{i \in F} a_{i} .
$$

Given a facet $F \in \Gamma_{n, r}$, we will refer to the sum

$$
\alpha(F, \mu)=\sum_{i \in F} a_{i}
$$

as the amount of $\mu$ covered by $F$. With this terminology, in order to calculate $f_{n, r}(\mu)$, we need to use $r$ disjoint edges in $\Gamma_{n}$ to cover as much of $\mu$ as possible.

Lemma 2.7 For any monomial $\mu \in A_{n}$ and $0 \leq r \leq k$, we have:

$f_{n, r}(\mu)=\sum_{j=r}^{k}(j+1-r) \operatorname{deg}_{j}(\mu) \quad$ and $\quad \operatorname{deg}_{r}(\mu)=\left(f_{n, r}-2 f_{n, r+1}+f_{n, r+2}\right)(\mu)$,

where $f_{n, j}=0$ for $j>k$.

Proof Consider the smallest entry $a$ in the last row of Conca's tableau for $\mu$. Since $a$ or $a-1$ must appear in each preceding row, we see that covering $\{a-1, a\}$ with an edge from $\Gamma_{n}$ will maximize the amount covered. Moreover, the rows are $<1$-chains, so we can cover exactly one entry from each row. In other words, the number of boxes in the first column of the tableau is equal to

$$
\max _{F \in \Gamma_{n}} \sum_{i \in F} a_{i}
$$

Therefore, $f_{n, 1}(\mu)$ is equal to the number of boxes that do not lie in the first column.

Now we remove the boxes with entries $a$ or $a-1$ from the tableau and repeat this argument for what remains. It follows that $f_{n, r}(\mu)$ is equal to the number of boxes of the tableau that do not lie in the first $r$ columns. Subtracting $r$ boxes from each row and adding up what remains, we find that

$$
f_{n, r}(\mu)=\sum_{j=r}^{k}(j+1-r) \operatorname{deg}_{j}(\mu) .
$$

Moreover, $\operatorname{deg}_{r}(\mu)$ is equal to the difference between the number of boxes in the $(r+1)$ th column and the $(r+2)$ th column. Therefore,

$$
\begin{aligned}
\operatorname{deg}_{r}(\mu) & =\left(f_{n, r}(\mu)-f_{n, r+1}(\mu)\right)-\left(f_{n, r+1}(\mu)-f_{n, r+2}(\mu)\right) \\
& =f_{n, r}(\mu)-2 f_{n, r+1}(\mu)+f_{n, r+2}(\mu),
\end{aligned}
$$

as desired. 
Example 2.8 Consider the monomial $\mu=z_{0}^{2} z_{1}^{4} z_{2}^{3} z_{3} z_{4} z_{5}^{4} \in A_{5}$ (15). Conca's decomposition for $\mu$ yields the tableau

\begin{tabular}{|l|l|l|}
\hline 0 & 2 & 4 \\
\hline 0 & 2 & 5 \\
\hline 1 & 3 & 5 \\
\hline 1 & 5 & \multicolumn{1}{|}{5} \\
\cline { 1 - 1 } 1 & 5 & \multicolumn{1}{|c}{} \\
\cline { 1 - 1 } 1 & \multicolumn{2}{|c}{} \\
\cline { 1 - 1 } 2 & \multicolumn{2}{|c}{} \\
\cline { 1 - 1 } & &
\end{tabular}

Note that $f_{5,0}(\mu)=\operatorname{deg}(\mu)=15$. First, we cover $\{1,2\}$, which removes seven boxes, so $f_{5,1}(\mu)=15-7=8$. Next, we cover $\{4,5\}$, which removes five boxes, so $f_{5,2}(\mu)=8-5=3$. Counting the number of rows of each size, we see that

$$
\operatorname{deg}_{0}(\mu)=2=15-2(8)+3, \quad \operatorname{deg}_{1}(\mu)=2=8-2(3), \quad \operatorname{deg}_{2}(\mu)=3,
$$

as expected.

\section{Structure of the level sets}

We have the following "tropical" decomposition of $A_{n}(m)$ :

$$
Q_{n}\left(d_{0}, \ldots, d_{k}\right)=\left\{\mu \in A_{n} \mid f_{n, r}(\mu)=\sum_{j=r}^{k}(j+1-r) d_{j} \text { for each } 0 \leq r \leq k\right\},
$$

where

$$
m=\sum_{j=0}^{k} d_{j}(j+1)
$$

Note that each $f_{n, r}$ is a piecewise-linear function from $\mathbb{R}^{n+1} \rightarrow \mathbb{R}$. It follows that $Q_{n}\left(d_{0}, \ldots, d_{k}\right)$ can be visualized as the set of integer lattice points in $\mathbb{R}_{\geq 0}^{n+1}$ that lie in the intersection of the given level sets:

$$
\bigcap_{0 \leq r \leq k}\left\{\left(a_{0}, \ldots, a_{n}\right) \mid f_{n, r}\left(a_{0}, \ldots, a_{n}\right)=(j+1-r) d_{j}\right\} .
$$

In fact, since $f_{n, 0}=m$, we can actually visualize these posets inside $\mathbb{R}^{n}$.

Example 3.1 We embed $A_{3}(m) \subset \mathbb{R}^{3}$ by drawing the covering relations along the three axes as follows:
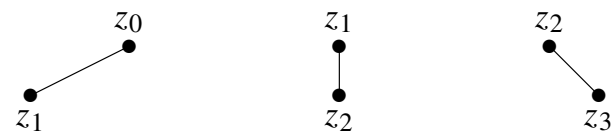
Below we have drawn the Hasse diagram of $A_{3}(4) \subset \mathbb{Z}_{\geq 0}^{3}$ along with its tropical decomposition into three subposets:
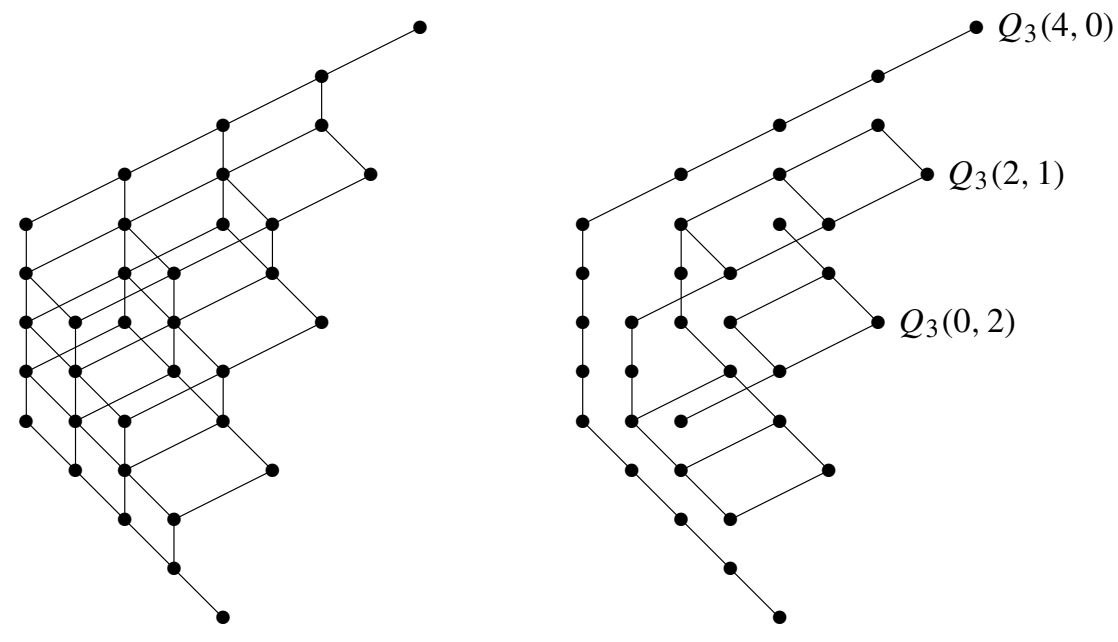

Proposition 3.2 $Q_{n}\left(d_{0}, \ldots, d_{k}\right)$ is a rank-symmetric, rank-unimodal, and centered subposet of $A_{n}(m)$.

Proof Since $\Gamma_{n}$ has an involution which sends $i$ to $n-i$, we see that $I_{n, r}$ and $f_{n, r}$ are invariant under the automorphism $\tau_{n}$ of $A_{n}$ that sends each $z_{i}$ to $z_{n-i}$. It follows that $Q_{n}\left(d_{0}, \ldots, d_{k}\right)$ carries a rank-flipping involution, which implies that it is a ranksymmetric, centered subposet of $A_{n}(m)$.

To prove the rank-unimodality of $Q_{n}\left(d_{0}, \ldots, d_{k}\right)$, let $J_{n, r}$ denote the ideal generated by maximal minors of the Hankel matrix $H_{n, r}$. Since all of the ideals involved are $\mathfrak{s l}_{2} \mathbb{C}$-invariant, the multigraded components of the algebra

$$
\operatorname{gr}_{J_{n, k}} \cdots \operatorname{gr}_{J_{n, 1}} A_{n}
$$

are finite-dimensional $\mathfrak{s l}_{2} \mathbb{C}$-representations. In particular, the dimensions of the weight spaces of each multigraded component form a unimodal sequence. Since the algebra

$$
\operatorname{gr}_{I_{n, k}} \cdots \operatorname{gr}_{I_{n, 1}} A_{n}
$$

is obtained by Gröbner degeneration, the dimensions of the weight spaces remain the same, and therefore the rank sizes of $Q_{n}\left(d_{0}, \ldots, d_{k}\right)$ form a unimodal sequence.

Note that $I_{n, r}$ has a unique minimal generator of highest (resp. lowest) weight, namely:

$$
\mu_{n, r}=z_{0} z_{2} \cdots z_{2 r} \quad\left(\text { resp. } v_{n, r}=\tau_{n}\left(\mu_{n, r}\right)=z_{n} z_{n-2} \cdots z_{n-2 r}\right) .
$$


It follows that $Q_{n}\left(d_{0}, \ldots, d_{k}\right)$ has a unique monomial of highest (resp. lowest) weight, namely:

$$
\mu_{n}\left(d_{0}, \ldots, d_{k}\right)=\prod_{j=0}^{k} \mu_{n, j}^{d_{j}} \quad\left(\operatorname{resp} . v_{n}\left(d_{0}, \ldots, d_{k}\right)=\prod_{j=0}^{k} v_{n, j}^{d_{j}}\right) .
$$

We can now state the embedding property of the $Q$-posets.

Lemma 3.3 Let $d_{0}, \ldots, d_{k} \geq 0$, and let $d=d_{0}+\cdots+d_{k}$. Then multiplication by $z_{n}^{d}$ induces an embedding of posets,

$$
z_{n}^{d}: Q_{n-2}\left(d_{1}, \ldots, d_{k}\right) \rightarrow Q_{n}\left(d_{0}, \ldots, d_{k}\right),
$$

which sends $v_{n-2}\left(d_{1}, \ldots, d_{k}\right)$ to $v_{n}\left(d_{0}, \ldots, d_{k}\right)$.

Proof Since $z_{n} v_{n-2, r}=v_{n, r}$, we see that

$$
z_{n}^{d} v_{n-2}\left(d_{1}, \ldots, d_{k}\right)=z_{n}^{d_{0}} \prod_{j=1}^{k} z_{n}^{d_{j}} v_{n-2, j}^{d_{j}}=\prod_{j=0}^{k} v_{n, j}^{d_{j}}=v_{n}\left(d_{0}, \ldots, d_{k}\right) .
$$

Let $\mu \in Q_{n-2}\left(d_{1}, \ldots, d_{k}\right)$. One can show that Conca's tableau for $z_{n}^{d} \mu$ is obtained from Conca's tableau for $\mu$ by adding a box with entry $n$ to the end of each row (along with $d_{0}$ extra rows of size one, each with entry $n$ ). We give a second proof below in terms of tropical polynomials.

Recall that

$$
\left(f_{n, 0}-f_{n, 1}\right)\left(a_{0}, \ldots, a_{n}\right)=\max _{0 \leq j \leq n-1}\left(a_{j}+a_{j+1}\right) .
$$

In the monomial $z_{n}^{d} \mu$, the sum of the exponents of $z_{n-1}$ and $z_{n}$ is equal to $d$, and

$$
d \geq d_{1}+\cdots+d_{k}=f_{n-2,0}(\mu)-f_{n-2,1}(\mu) .
$$

In other words, the edge $\{n-1, n\}$ will cover at least as much of $z_{n}^{d} \mu$ as any other edge in $\Gamma_{n}$.

Now we claim that, for each $1 \leq r \leq k$,

$$
f_{n, r}\left(z_{n}^{d} \mu\right)=f_{n-2, r-1}(\mu) .
$$

Recall that the calculation of $f_{n, r}$ involves all $r$-fold disjoint unions of edges in $\Gamma_{n}$. We can split up this calculation into two cases: the $n$th vertex is either covered or uncovered. If the $n$th vertex is covered, then so is the $(n-1)$ th vertex, which leaves $r-1$ edges for the vertices $0, \ldots, n-2$. In other words, for $z_{n}^{d} \mu$, we have

$$
\max _{n \in F \in \Gamma_{n, r}} \sum_{i \in F} a_{i}=d+\max _{F \in \Gamma_{n-2, r-1}} \sum_{i \in F} a_{i}=d+\operatorname{deg}(\mu)-f_{n-2, r-1}(\mu) .
$$


On the other hand, if the $n$th vertex is uncovered, choose $r-1$ pairwise disjoint edges from $\Gamma_{n-2}$ and then arbitrarily choose the $r$ th edge from $\Gamma_{n-1}$. Since the exponent of $z_{n-1}$ in $z_{n}^{d} \mu$ is zero, we get the following inequalities:

$$
\begin{aligned}
\max _{n \notin F \in \Gamma_{n, r}} \sum_{i \in F} a_{i} & \leq \max _{0 \leq j \leq n-2}\left(a_{j}+a_{j+1}\right)+\max _{F \in \Gamma_{n-2, r-1}} \sum_{i \in F} a_{i} \\
& \leq d+\operatorname{deg}(\mu)-f_{n-2, r-1}(\mu) .
\end{aligned}
$$

Therefore,

$$
\begin{aligned}
f_{n, r}\left(z_{n}^{d} \mu\right) & =\operatorname{deg}\left(z_{n}^{d} \mu\right)-\max _{F \in \Gamma_{n, r}} \sum_{i \in F} a_{i} \\
& =d+\operatorname{deg}(\mu)-\max \left(\max _{n \in F \in \Gamma_{n, r}} \sum_{i \in F} a_{i}, \max _{n \notin F \in \Gamma_{n, r}} \sum_{i \in F} a_{i}\right) \\
& =d+\operatorname{deg}(\mu)-d-\operatorname{deg}(\mu)+f_{n-2, r-1}(\mu) \\
& =f_{n-2, r-1}(\mu) .
\end{aligned}
$$

It follows that, for $1 \leq r \leq k$,

$$
\begin{aligned}
\operatorname{deg}_{r}\left(z_{n}^{d} \mu\right) & =\left(f_{n, r}-2 f_{n, r+1}+f_{n, r+2}\right)\left(z_{n}^{d} \mu\right) \\
& =\left(f_{n, r-1}-2 f_{n, r}+f_{n, r+1}\right)(\mu) \\
& =\operatorname{deg}_{r-1}(\mu) .
\end{aligned}
$$

The only difference occurs at $r=0$ :

$$
\begin{aligned}
\operatorname{deg}_{0}\left(z_{n}^{d} \mu\right) & =\left(f_{n, 0}-2 f_{n, 1}+f_{n, 2}\right)\left(z_{n}^{d} \mu\right) \\
& =d+\left(f_{n-2,0}-2 f_{n-2,0}+f_{n-2,1}\right)(\mu) \\
& =d-\left(f_{n-2,0}-f_{n-2,1}\right)(\mu) \\
& =d-\left(d_{1}+\cdots+d_{k}\right) \\
& =d_{0}
\end{aligned}
$$

Therefore, we have shown that multiplication by $z_{n}^{d}$ defines a map

$$
Q_{n-2}\left(d_{1}, \ldots, d_{k}\right) \rightarrow Q_{n}\left(d_{0}, \ldots, d_{k}\right)
$$

Since multiplication by a fixed element is injective and preserves the partial order, this map is an embedding of posets.

Remark 3.4 Composing the above embedding with the rank-flipping involution on both sides, we get another embedding,

$$
\tau_{n} z_{n}^{d} \tau_{n-2}: Q_{n-2}\left(d_{1}, \ldots, d_{k}\right) \rightarrow Q_{n}\left(d_{0}, \ldots, d_{k}\right)
$$


which sends $\mu_{n-2}\left(d_{1}, \ldots, d_{k}\right)$ to $\mu_{n}\left(d_{0}, \ldots, d_{k}\right)$. We can express this embedding as the composition of the homomorphism $\kappa_{n}: A_{n-2} \rightarrow A_{n}$ that sends each $z_{i} \mapsto z_{i+2}$, followed by multiplication by $z_{0}^{d}$. Indeed, for any minimal generator $z_{i_{0}} \ldots z_{i_{r}}$ of $I_{n-2, r}$ we have:

$$
\begin{aligned}
\tau_{n} z_{n} \tau_{n-2}\left(z_{i_{0}} \cdots z_{i_{r}}\right) & =\tau_{n}\left(z_{n} z_{n-2-i_{0}} \cdots z_{n-2-i_{r}}\right) \\
& =z_{0} z_{i_{0}+2} \cdots z_{i_{r}+2} \\
& =z_{0} \kappa_{n}\left(z_{i_{0}} \cdots z_{i_{r}}\right)
\end{aligned}
$$

from which the general formula follows.

Remark 3.5 The images of the above embeddings can be written down in elementary terms as well:

$$
\begin{aligned}
z_{0}^{d} \kappa_{n} Q_{n-2}\left(d_{1}, \ldots, d_{k}\right) & =\left\{\mu \in Q_{n}\left(d_{0}, \ldots, d_{k}\right) \mid a_{0}=\max _{0 \leq j \leq n-1}\left(a_{j}+a_{j+1}\right)\right\}, \\
z_{n}^{d} Q_{n-2}\left(d_{1}, \ldots, d_{k}\right) & =\left\{\mu \in Q_{n}\left(d_{0}, \ldots, d_{k}\right) \mid a_{n}=\max _{0 \leq j \leq n-1}\left(a_{j}+a_{j+1}\right)\right\} .
\end{aligned}
$$

These equalities follow immediately from the fact that covering $\{0,1\}$ (resp. $\{n-$ $1, n\}$ ) will cover the maximum possible amount in each such monomial.

\section{The raising and lowering algorithm}

Recall that the Hasse diagram of $A_{n}(m)$ is equipped with an edge coloring by $\{1, \ldots, n\}$, where the $i$ th color corresponds to the following covering relation:

$$
\left(a_{0}, \ldots, a_{n}\right) \rightarrow\left(a_{0}, \ldots a_{i-1}-1, a_{i}+1, \ldots, a_{n}\right)
$$

Let $C$ be a saturated chain in $A_{n}(m)$. We obtain a sequence of colors $\left(c_{1}, \ldots, c_{t}\right)$ by reading the covering relations in $C$ from highest to lowest weight. We say that a saturated chain $C$ is monotonic if $c_{1} \leq \cdots \leq c_{t}$.

A pair of consecutive entries $\left(a_{i}, a_{i+1}\right)$ of $\left(a_{0}, \ldots, a_{n}\right)$ is called a maximal pair if

$$
a_{i}+a_{i+1}=\max _{0 \leq j \leq n-1}\left(a_{j}+a_{j+1}\right)
$$

Let $\mu=z_{0}^{a_{0}} \cdots z_{n}^{a_{n}} \in Q_{n}\left(d_{0}, \ldots, d_{k}\right)$. The following "right-moving" algorithm produces a monotonic saturated chain between $\mu$ and an element of $z_{n}^{d} Q_{n-2}\left(d_{1}, \ldots, d_{k}\right)$ :

1. Let $\left(a_{i}, a_{i+1}\right)$ be a maximal pair of $\left(a_{0}, \ldots, a_{n}\right)$. If $i=n-1$, apply the $n$th color $a_{n-1}$ times and end the chain.

2. If $i<n-1$, apply the $(i+1)$ th color $\left(a_{i}-a_{i+2}\right)$ times, then go back to the first step and choose the maximal pair $\left(a_{i+1}, a_{i+2}\right)$.

The corresponding "left-moving" algorithm produces a monotonic saturated chain between $\mu$ and an element of $z_{0}^{d} \kappa_{n} Q_{n-2}\left(d_{1}, \ldots, d_{k}\right)$ : 
1. Let $\left(a_{i-1}, a_{i}\right)$ be a maximal pair of $\left(a_{0}, \ldots, a_{n}\right)$. If $i=1$, apply the inverse of the first color $a_{1}$ times and end the chain.

2. If $i>1$, apply the inverse of the $i$ th color $\left(a_{i}-a_{i-2}\right)$ times, then go back to the first step and choose the maximal pair $\left(a_{i-2}, a_{i-1}\right)$.

The left transversal chain of $\mu$ is constructed as follows. We begin with the leftmost maximal pair in $\left(a_{0}, \ldots, a_{n}\right)$ and run both the left-moving and right-moving algorithms. The concatenation of the two resulting chains is a single monotonic saturated chain passing through $\mu$ which runs between

$$
z_{0}^{d} \kappa_{n} Q_{n-2}\left(d_{1}, \ldots, d_{k}\right) \quad \text { and } \quad z_{n}^{d} Q_{n-2}\left(d_{1}, \ldots, d_{k}\right) .
$$

Similarly, the right transversal chain of $\mu$ is constructed by applying the same procedure starting with the rightmost maximal pair in $\left(a_{0}, \ldots, a_{n}\right)$.

Example 4.1 Consider the monomial $\mu=z_{0} z_{1} z_{2} z_{4} z_{5} \in A_{5}(5)$. We easily calculate that $f_{5,1}(\mu)=3$ and $f_{5,2}(\mu)=1$, so $\mu \in Q_{5}(0,1,1)$. The corresponding lattice vector is $\left(a_{0}, \ldots, a_{5}\right)=(1,1,1,0,1,1)$, which we can visualize as five indistinguishable balls distributed among six labeled urns. The following diagrams represent the calculation of the left and right transversal chains of $\mu$ :

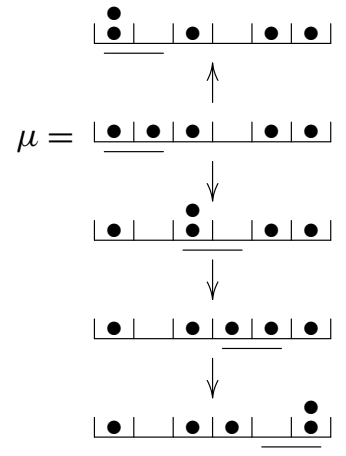

Left transversal chain

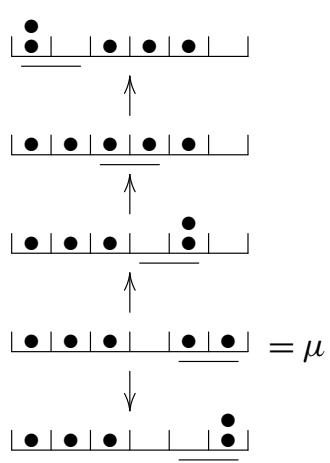

Right transversal chain

Upward arrows denote left moves, downward arrows denote right moves, and covered maximal pairs are underlined. We have chosen not to draw the steps where no balls are moved and the only change is the movement of the covering edge.

Theorem 4.2 Let $n, d_{0}, \ldots, d_{k} \geq 0$. The transversal chains provide a vertex covering of $Q_{n}\left(d_{0}, \ldots, d_{k}\right)$ by monotonic saturated chains. If $d_{0}>0$, then this covering is actually a decomposition.

Proof For the first statement, the only thing to check is that the algorithm stays within $Q_{n}\left(d_{0}, \ldots, d_{k}\right)$ at each step. We claim that if $\left(a_{i}, a_{i+1}\right)$ is a maximal pair of $\left(a_{0}, \ldots, a_{n}\right)$ and $a_{i}>a_{i+2}$, then

$$
f_{n, r}\left(a_{0}, \ldots, a_{n}\right)=f_{n, r}\left(a_{0}, \ldots, a_{i}-1, a_{i+1}+1, \ldots, a_{n}\right)
$$


for all $0 \leq r \leq k$. To prove this, let us deal with each possible case in turn. Let $\mu^{\prime}$ denote the monomial obtained by applying the $(i+1)$ th color to $\mu$. Recall that

$$
f_{n, r}(\mu)=\min _{F \in \Gamma_{n, r}} \alpha(\bar{F}, \mu) .
$$

If $F \in \Gamma_{n, r}$ is a facet such that $\{i, i+1\} \subset F$, then $\alpha\left(\bar{F}, \mu^{\prime}\right)=\alpha(\bar{F}, \mu)$. If $i \in F$ and $i+1 \notin F$, then $\alpha\left(\bar{F}, \mu^{\prime}\right)=\alpha(\bar{F}, \mu)+1$, which means that it is irrelevant for calculating the minimum over such sums. The only other possibility is that $i \notin F$ and $i+1 \in F$, which means that $i+2 \in F$ as well. In this case, since $a_{i}>a_{i+2}$, we see that $\alpha(\bar{F}, \mu)>f_{n, r}(\mu)$, because moving the edge to the left by one step and covering $\{i, i+1\}$ would strictly decrease the sum. Therefore, $\alpha\left(\bar{F}, \mu^{\prime}\right)=\alpha(\bar{F}, \mu)-$ $1 \geq f_{n, r}(\mu)$, and the overall minimization will be unchanged.

For the second statement, if $d_{0}>0$, then Conca's tableau for $\mu$ has at least one row of size one. If the entry in the last row is 0 (resp. $n)$, then $\left(a_{0}, a_{1}\right)\left(\operatorname{resp} .\left(a_{n-1}, a_{n}\right)\right)$ is the unique maximal pair. Otherwise, if the entry in the last row is $0<i<n$, then $\left(a_{i-1}, a_{i}\right)$ is a maximal pair. The only other possible maximal pair is $\left(a_{i}, a_{i+1}\right)$, which can only happen if $a_{i-1}=a_{i+1}$. In this case, the algorithm will only move the covering edge, so there is essentially a unique starting maximal pair. It follows that the left and right transversal chains of $\mu$ coincide and the transversal chains are necessarily disjoint from each other by the uniqueness of each step of the algorithm. Therefore, if $d_{0}>0$, we obtain a monotonic saturated chain decomposition of $Q_{n}\left(d_{0}, \ldots, d_{k}\right)$.

Corollary 4.3 If $Q_{n-2}\left(d_{1}, \ldots, d_{k}\right)$ has a symmetric chain decomposition and

$$
1+2 d_{0} \geq \sum_{j=1}^{k} d_{j}(n-2 j) j,
$$

then $Q_{n}\left(d_{0}, \ldots, d_{k}\right)$ has a symmetric chain decomposition.

Proof Suppose we have a decomposition

$$
Q_{n-2}\left(d_{1}, \ldots, d_{k}\right)=C_{1} \sqcup \cdots \sqcup C_{t}
$$

where each $C_{i}$ is a rank-symmetric, saturated chain. Let $\mu_{0}$ be a monomial in $C_{i}$ for some $i$. Let $d=d_{0}+\cdots+d_{k}$ and consider the monomial

$$
\mu=z_{0}^{a_{0}} \ldots z_{n}^{a_{n}}=\tau_{n} z_{n}^{d} \tau_{n-2} \mu_{0}=z_{0}^{d} \kappa_{n} \mu_{0} \in Q_{n}\left(d_{0}, \ldots, d_{k}\right) .
$$

Let us calculate the left transversal chain of $\mu$. The leftmost maximal pair is $\left(a_{0}, a_{1}\right)$ and $a_{1}=0$, so the first few steps of the algorithm look like

$$
\begin{gathered}
\left(a_{0}, 0, a_{2}, a_{3}, \ldots, a_{n}\right), \\
\left(a_{2}, a_{0}-a_{2}, a_{2}, a_{3}, \ldots, a_{n}\right), \\
\left(a_{2}, a_{3}, a_{0}-a_{3}, a_{3}, \ldots, a_{n}\right),
\end{gathered}
$$




$$
\begin{gathered}
\left(a_{2}, a_{3}, \ldots, a_{n}, a_{0}-a_{n}, a_{n}\right), \\
\left(a_{2}, a_{3}, \ldots, a_{n}, 0, a_{0}\right) .
\end{gathered}
$$

We see that the $a_{0}$ term travels to the right while all the other entries shift to the left by two spots. In other words, the left transversal chain of $z_{0}^{d} \kappa_{n} \mu_{0}$ in $Q_{n}\left(d_{0}, \ldots, d_{k}\right)$ has lowest weight monomial $z_{n}^{d} \mu_{0}$. The key point is that, for $d_{0}>0$, we obtain a decomposition

$$
Q_{n}\left(d_{0}, \ldots, d_{k}\right)=R_{1} \sqcup \cdots \sqcup R_{t}
$$

where $R_{i}$ is the induced subposet that contains the chains $z_{0}^{d} \kappa_{n} C_{i}$ and $z_{n}^{d} C_{i}$, as well as all the transversal chains between them. Note that $R_{i}$ also contains $d_{0}+1$ translates of $z_{0}^{d} \kappa_{n} C_{i}$ (resp. $z_{n}^{d} C_{i}$ ), namely

$$
z_{0}^{d-j} z_{1}^{j} \kappa_{n} C_{i} \quad\left(\text { resp. } z_{n-1}^{j} z_{n}^{d-j} C_{i}\right) \quad \text { for } 0 \leq j \leq d_{0}
$$

The general structure of $R_{i}$ can be represented as follows:

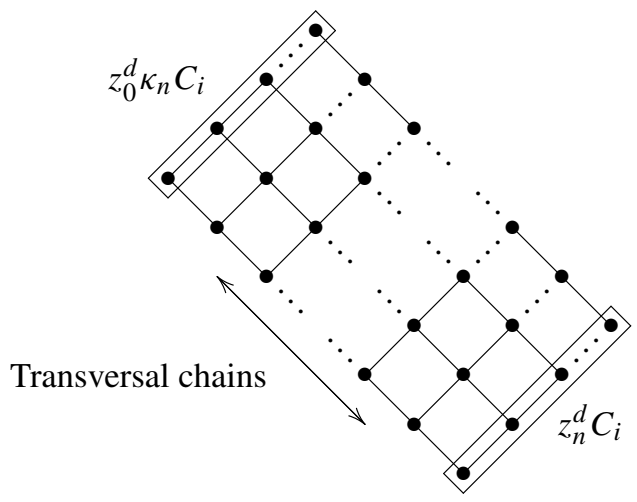

Now the boundary of this rectangle can be thought of as a disjoint union of a pair of symmetric saturated chains (in two different ways). If we remove such a pair of symmetric chains, we are left with a smaller rectangle. We can continue this process as long as we are guaranteed that the boundary of what remains is connected. At each step, the length of $C_{i}$ is truncated by two, and the length of each transversal chain is truncated by four. Therefore, for $R_{i}$ to have a symmetric chain decomposition, it is sufficient that $2 d_{0}+1$ is greater than or equal to the number of edges in a maximal chain of $Q_{n-2}\left(d_{1}, \ldots, d_{k}\right)$. We may assume that $C_{1}$ is the chain containing the unique highest weight monomial $\mu_{n-2}\left(d_{1}, \ldots, d_{k}\right)$ (resp. lowest weight monomial $\left.v_{n-2}\left(d_{1}, \ldots, d_{k}\right)\right)$ in $Q_{n-2}\left(d_{1}, \ldots, d_{k}\right)$. Note that the number of edges in any chain of $Q_{n}\left(d_{0}, \ldots, d_{k}\right)$ of maximal length is equal to the weight of $\mu_{n}\left(d_{0}, \ldots, d_{k}\right)$, and 


$$
\begin{aligned}
\mathrm{wt}\left(\mu_{n}\left(d_{0}, \ldots, d_{k}\right)\right) & =\sum_{j=0}^{k} d_{j} \mathrm{wt}\left(\mu_{n, j}\right) \\
& =\sum_{j=0}^{k} d_{j} \sum_{i=0}^{j}(n-4 i) \\
& =\sum_{j=0}^{k} d_{j}(n(j+1)-2 j(j+1)) \\
& =\sum_{j=0}^{k} d_{j}(n-2 j)(j+1) .
\end{aligned}
$$

Therefore, the number of edges in $C_{1}$ is equal to

$$
\sum_{j=0}^{k-1} d_{j+1}(n-2-2 j)(j+1)=\sum_{j=1}^{k} d_{j}(n-2 j) j .
$$

We conclude that if

$$
1+2 d_{0} \geq \sum_{j=1}^{k} d_{j}(n-2 j) j
$$

then $Q_{n}\left(d_{0}, \ldots, d_{k}\right)$ has a symmetric chain decomposition.

We can now define the generic part of $L(m, n)$. We say that $\left(d_{0}, \ldots, d_{k}\right) \in \mathbb{Z}_{\geq 0}^{k+1}$ is $n$-stable if

$$
1+2 d_{0} \geq \sum_{j=1}^{k} d_{j}(n-2 j) j
$$

and we say that $\left(d_{0}, \ldots, d_{k}\right) \in \mathbb{Z}_{\geq 0}^{k+1}$ is generic if $\left(d_{r}, \ldots, d_{k}\right)$ is $(n-2 r)$-stable for each $0 \leq r \leq k$. Now we define

$$
L(m, n)^{\text {gen }}=\bigsqcup_{\substack{\left(d_{0}, \ldots, d_{k}\right) \\ \text { generic }}} Q_{n}\left(d_{0}, \ldots, d_{k}\right)
$$

Let $L(m, n)^{\text {sing }}$ denote the complement of $L(m, n)^{\text {gen }}$ in $L(m, n)$, equipped with the induced partial order. Then Corollary 4.3 implies that there is a decomposition of $L(m, n)$ into centered, rank-symmetric, and rank-unimodal subposets:

$$
L(m, n)=L(m, n)^{\mathrm{gen}} \sqcup L(m, n)^{\mathrm{sing}}
$$

such that $L(m, n)^{\text {gen }}$ is a symmetric chain order. 
Conjecture 4.4 We conjecture that $Q_{n}\left(d_{0}, \ldots, d_{k}\right)$ is a symmetric chain order for all $n, d_{0}, \ldots, d_{k} \geq 0$. This statement is strictly stronger than the statement that $L(m, n)$ is a symmetric chain order for all $m, n \geq 0$ since there are examples of $m$ and $n$ where symmetric chain decompositions of $L(m, n)$ do not necessarily respect the tropical decomposition.

Remark 4.5 The inequality presented in the theorem is not a necessary condition for $Q_{n}\left(d_{0}, \ldots, d_{k}\right)$ to have a symmetric chain decomposition. Indeed, the argument will produce a symmetric chain decomposition for $R_{i}$ as long as we can successively peel off two boundary chains at a time and leave a rectangle with connected boundary.

Corollary 4.6 $L(m, n)$ is a symmetric chain order if $\min (m, n) \leq 4$.

Proof First note that if $\min (m, n) \leq 2$, then $L(m, n)=L(m, n)^{\text {gen }}$, so we are done by Corollary 4.3. By the way, since $Q_{2}(0,1)=\left\{z_{0} z_{2}\right\}$, we have an isomorphism of ranked posets $Q_{2}\left(d_{0}, d_{1}\right) \simeq Q_{2}\left(d_{0}, 0\right)$, which consists of a single symmetric chain of size $2 d_{0}+1$. It follows that our decomposition

$$
A_{2}(m)=\bigsqcup_{j=0}^{\lfloor m / 2\rfloor} Q_{2}(m-2 j, j) \simeq \bigsqcup_{j=0}^{\lfloor m / 2\rfloor} Q_{2}(m-2 j, 0)
$$

is already a symmetric chain decomposition.

If $n=3$, then we have

$$
A_{3}(m)=\bigsqcup_{j=0}^{\lfloor m / 2\rfloor} Q_{3}(m-2 j, j)
$$

If $d_{0}=0$, then $m=2 j$, and there is an isomorphism of ranked posets:

$$
\begin{aligned}
Q_{3}(0, m / 2) & \simeq A_{2}(m / 2) \\
\left(z_{0} z_{2}\right)^{a_{02}}\left(z_{0} z_{3}\right)^{a_{03}}\left(z_{1} z_{3}\right)^{a_{13}} & \mapsto\left(a_{02}, a_{03}, a_{13}\right),
\end{aligned}
$$

so $Q_{3}(0, m / 2)$ is a symmetric chain order. On the other hand, if $d_{0}>0$, then the two embedded copies of $Q_{1}(j) \subset Q_{3}(m-2 j, j)$ are both saturated chains of size $(j+1)$, so $Q_{3}(m-2 j, j)$ is a rectangular poset of the type discussed in the proof of Corollary 4.3. The top copy of $Q_{1}(j)$ consists of monomials of the form

$$
z_{0}^{d_{0}}\left(z_{0} z_{2}\right)^{d_{1}-j}\left(z_{0} z_{3}\right)^{j}
$$

Since the first color (which changes a single $z_{0}$ to a $\left.z_{1}\right)$ can be applied $\left(d_{0}+j\right)$ times to the $j$ th element, we see that there will be sufficient translates of $Q_{1}(j)$ in $Q_{3}(m-2 j, j)$ for the argument of Corollary 4.3 to work. 
If $n=4$, then we have

$$
A_{4}(m)=\bigsqcup_{d_{0}+2 d_{1}+3 d_{2}=m} Q_{4}\left(d_{0}, d_{1}, d_{2}\right) .
$$

Note that $Q_{4}(0,0,1)=\left\{z_{0} z_{2} z_{4}\right\}$, so

$$
Q_{4}\left(d_{0}, d_{1}, d_{2}\right) \simeq Q_{4}\left(d_{0}, d_{1}, 0\right) .
$$

It follows that

$$
A_{4}(m) \simeq \bigsqcup_{j=0}^{\lfloor m / 2\rfloor} Q_{4}(m-2 j, j, 0)
$$

If $d_{0}=0$, we have an isomorphism of ranked posets,

$$
\begin{aligned}
Q_{4}(0, m / 2,0) & \simeq A_{2}(m) \\
\left(z_{i} z_{j}\right)^{a_{i j}} & \mapsto\left(2 a_{02}+a_{03}+a_{13}, a_{03}+2 a_{04}+a_{14}, a_{13}+a_{14}+2 a_{24}\right) .
\end{aligned}
$$

If $d_{0}>0$, we have the two copies $Q_{2}(j, 0) \subset Q_{4}(m-2 j, j, 0)$, which are saturated chains of size $(2 j+1)$. The top copy consists of monomials of two types,

$$
z_{0}^{d_{0}}\left(z_{0} z_{2}\right)^{d_{1}-j}\left(z_{0} z_{3}\right)^{j} \quad \text { and } \quad z_{0}^{d_{0}}\left(z_{0} z_{3}\right)^{d_{1}-j}\left(z_{0} z_{4}\right)^{j}
$$

The first color can be applied $\left(d_{0}+j\right)$ times to elements of the first type, while the elements of the second type can receive $\left(d_{0}+d_{1}\right)$ applications of the first color followed by $j$ applications of the second color. Again, it follows that there will be sufficient translates of $Q_{2}(j, 0)$ available to apply the proof technique of Corollary 4.3.

Example 4.7 To see why the proof of Corollary 4.6 does not generalize, consider $Q_{5}(0,1,1) \subset L(5,5)^{\text {sing }}$. We draw the covering relations of $A_{5}(\mathrm{~m})$ along the five coordinate axes of $\mathbb{R}^{5}$ as follows:
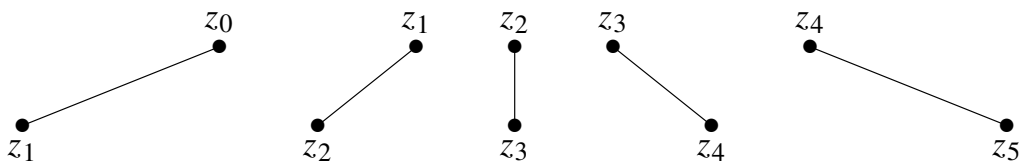
and we obtain the following embedding of $Q_{5}(0,1,1) \subset \mathbb{Z}_{\geq 0}^{5}$ :

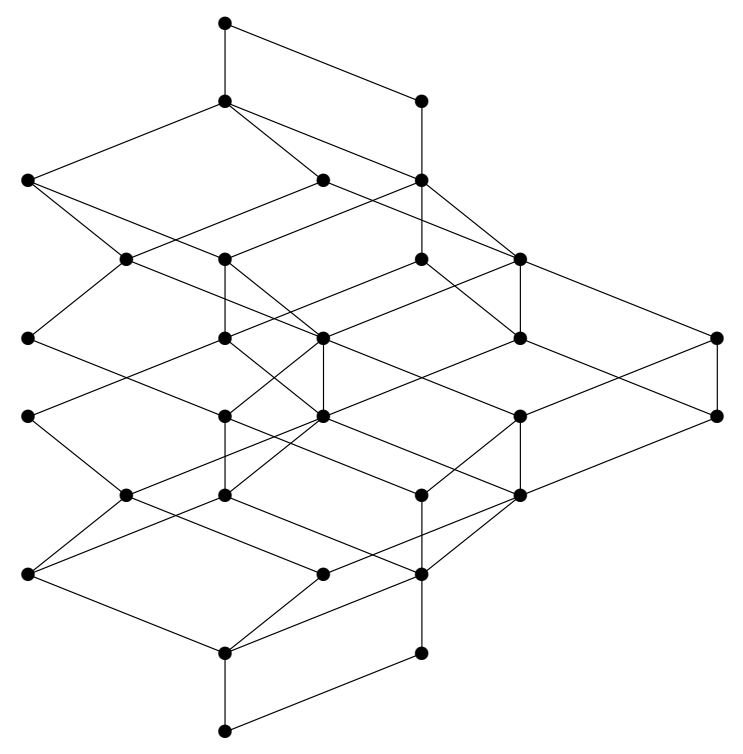

For $\min (m, n) \leq 4$ and $d_{0}=0$, we could identify the $Q$-posets in terms of posets encountered earlier. In this case, we find a poset containing overlapping copies of both $Q_{5}(0,1,0) \simeq L(2,3)$ and $Q_{5}(0,0,1) \simeq L(3,1)$. In such composite cases, it is not clear how to stitch the known symmetric chain decompositions together. Also, in general, when $d_{0}>0$, one has to choose a symmetric chain decomposition of $Q_{n-2}\left(d_{1}, \ldots, d_{k}\right)$ in order to obtain a rectangular decomposition of $Q_{n}\left(d_{0}, \ldots, d_{k}\right)$, and some choices may result in rectangles that are not symmetric chain orders.

Acknowledgements Thanks are due to Peter Magyar for telling me about Stanley's conjecture and for many enlightening conversations on a host of mathematical topics. I would also like to thank the Department of Mathematics at Michigan State University for their hospitality while this project was under way.

\section{References}

1. Conca, A.: Straightening law and powers of determinantal ideals of Hankel matrices. Adv. Math. 138, 263-292 (1998)

2. Lindström, B.: A partition of $L(3, n)$ into saturated chains. Eur. J. Comb. 1, 61-63 (1980)

3. O’Hara, K.: Unimodality of Gaussian coefficients: a constructive proof. J. Comb. Theory, Ser. A 53, 29-52 (1990)

4. Proctor, R.A.: Solution of two difficult combinatorial problems with linear algebra. Am. Math. Mon. 89, 721-734 (1982)

5. Riess, W.: Zwei Optimierungsprobleme auf Ordnungen. In: Arbeitsberichte des Institut für Mathematische Maschinen und Datenverarbeitung (Informatik), Bank II, Number, 5 Erlangen, pp. 50-57 (1978)

6. Stanley, R.: Weyl groups, the hard Lefschetz theorem, and the Sperner property. SIAM J. Algebr. Discrete Methods 1, 168-184 (1980) 
7. Sturmfels, B., Sullivant, S.: Combinatorial secant varieties. Pure Appl. Math. Q. 2(3), 867-891 (2006). Part 1

8. Sullivant, S.: Combinatorial symbolic powers. J. Algebra 319(1), 115-142 (2008)

9. West, D.: A symmetric chain decomposition of $L(4, n)$. Eur. J. Comb. 1, 379-383 (1980)

10. Zeilberger, D.: Kathy O'Hara's constructive proof of the unimodality of the Gaussian polynomials. Am. Math. Mon. 96, 590-602 (1989) 